\title{
Paleocanais no megaleque do rio Taquari: mapeamento e significado geomorfológico
}

\author{
Hiran Zani ${ }^{1} \&$ Mario Luis Assine ${ }^{2}$
}

\begin{abstract}
Resumo Este trabalho tem como objetivo apresentar o primeiro mapeamento sistemático da rede de paleocanais existente no megaleque do Taquari. Foram utilizados dados de sensoriamento coletados pelo sensor ASTER, que produz imagens com pixel de $15 \times 15 \mathrm{~m}$. Foram necessárias um total de 34 cenas, adquiridas entre os anos de 2001 a 2006. Estes dados foram processados através da técnica de realce por decorrelação, visando melhor identificação visual dos paleocanais. A etapa de mapeamento se iniciou com a sobreposição de uma quadrícula compatível com a articulação 1:50.000 do IBGE, a fim de sistematizar a extração das feições de interesse e diminuir a subjetividade inerente ao método de análise visual. Foram cartografados um total de $33.205 \mathrm{~km}$ de paleocanais. A densidade destas feições se mostrou relacionada com as idades relativas dos compartimentos geomorfológicos. As feições mapeadas preservam eventos de avulsão que ocorreram no megaleque, sugerindo que os de menor magnitude são mais frequentes.
\end{abstract}

Palavras-chave: avulsão, Pantanal, sensoriamento remoto, geomorfologia fluvial.

\begin{abstract}
Paleochannels on Taquari river megafan, Pantanal, Brazil: their mapping and geomorphological meaning. This paper presents the results of the first systematic mapping of the paleochannel network on the Taquari megafan (Pantanal, Brazil). We used remote sensing data collected by the ASTER sensor, which captures images with $15 \mathrm{~m}$ square pixels. A total of 34 scenes, acquired from 2001 - 2006, were used in the analysis. These data were processed using a decorrelation stretch technique in order to obtain a better visual identification of the paleochannels. The mapping procedure began with the overlay of a 1:50.000 IBGE articulation, in order to systematize the extraction of features and to reduce the subjectivity inherent to visual analysis methods. We mapped a total of $33,205 \mathrm{~km}$ of paleochannels. The density of these features is related to the relative ages of distinct geomorphological compartments, known as depositional lobes. The mapped features preserve avulsion events that occurred on the megafan. The results suggest that low-magnitude avulsions occur more frequently than larger events.
\end{abstract}

Keywords: avulsion, Pantanal wetland, remote sensing, fluvial geomorphology.

INTRODUÇÃO O megaleque do Taquari (Fig. 1) é descrito por muitos pesquisadores como unidade a geomorfológica mais evidente da Bacia Sedimentar do Pantanal (Braun 1977, Tricart 1982, Klammer 1982, Assine \& Soares 2004, Zani 2008, Zani et al. 2009a). Possui declividade média de $0.03^{\circ}$, distribuída em $49.000 \mathrm{~km}^{2}$ de área e ocupa um total de $35 \%$ da Bacia. Este baixo gradiente topográfico reduz a capacidade de transporte e erosão do rio Taquari, que após percorrer $150 \mathrm{~km}$ no Pantanal, assume padrão de drenagem distributário e deposita os sedimentos contidos no canal, dando origem a uma zona com elevadas taxas de sedimentação, que foi definida por Assine (2005) como lobo deposicional atual.

Assine (2003) observou que além do lobo deposicional atual, o megaleque do Taquari é composto por outros cinco lobos deposicionais, os quais não recebem mais sedimentos, denominados lobos deposicionais abandonados. Estes compartimentos refletem os distintos eventos de sedimentação que ocorreram nesta região do Pantanal, responsáveis pela morfologia atual do megaleque. Uma vasta rede de paleocanais está preservada na superfície e pode ser observada a partir imagens de satélite (e.g., Braun 1977, Assine 2005). Estas feições registram transformações recentes que ocorreram na paisagem, associadas com eventos de mudança do canal fluvial, denominados avulsão.

Embora alguns pesquisadores considerem que mudanças de grande magnitude dos canais fluviais são raramente observadas na escala de tempo da vida humana (Jones \& Hajek 2007), Padovani et al. (2001) documentaram a ocorrência de um evento de avulsão que transpôs a foz do rio Taquari em $60 \mathrm{~km}$. De acordo com Assine (2005), este processo se iniciou com o rompimento dos diques marginais no ano de 1990, até culminar com o abandono do antigo leito e estabelecimento de um novo canal no ano de 2000. Atualmente, outro fenômeno de avulsão está em curso na parte inferior do rio Taquari e poderá resultar em nova mudança do canal nos próximos anos. Neste contexto, o mapeamento sistemático dos paleocanais é de grande importância 


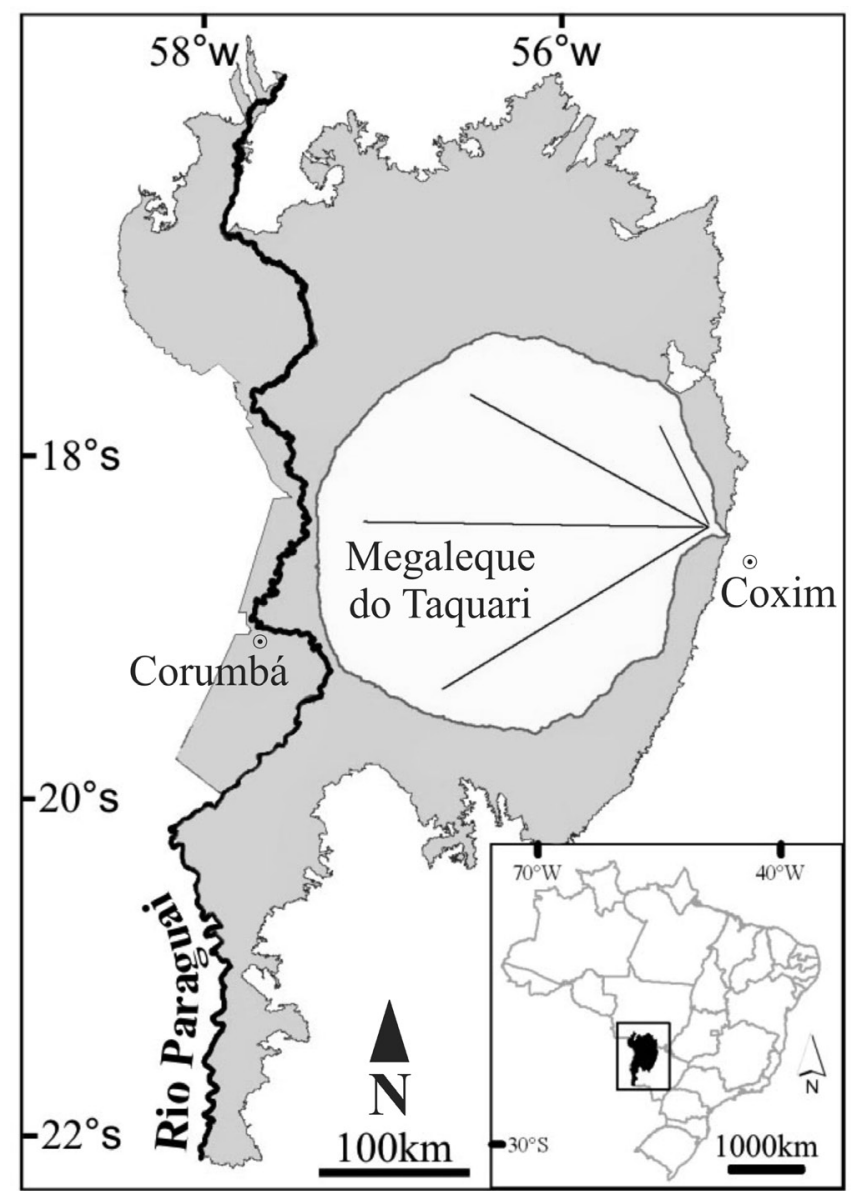

Figura 1 - Localização do megaleque do Taquari no Pantanal mato-grossense.

para a compreensão deste tipo de fenômeno e constitui uma etapa fundamental para se conhecer a dinâmica e evolução deste sistema deposicional.

Este artigo tem como objetivo apresentar o resultado do primeiro mapeamento sistemático dos paleocanais no megaleque do Taquari. A partir deste plano de informação, são feitas considerações sobre a geomorfologia do megaleque, com ênfase nos processos de avulsão e reconstituição dos eventos de sedimentação que ocorreram.

\section{PALEOCANAIS E SENSORIAMENTO REMO-}

TO Dados de sensoriamento remoto constituem a principal fonte de informação para identificação e mapeamento da geometria dos paleocanais (Ramasamy et al. 1991, Sinha 1996, Wray 2009). Possibilitam visualização sinóptica dos ambientes, fundamental para análise de extensas áreas, como o megaleque do Taquari. Além disso, técnicas de processamento digital de imagens e utilização de comprimentos de onda não restritos a faixa espectral do visível, permitem a extração de feições que são dificilmente observadas em campo.

Paleocanais são depósitos arenosos de antigos sistemas fluviais, que podem estar parcialmente ou totalmente soterrados por sedimentos mais recentes. Assim, sua identificação nas imagens orbitais está condicionada com o grau de preservação destas feições na superfície. No megaleque do Taquari observa-se a ocorrência de paleocanais em três estágios de conservação (Fig. 2): a) paleocanais com depósitos arenosos expostos; b) paleocanais parcialmente cobertos por vegetação rasteira; e c) paleocanais cobertos por vegetação arbustiva. Na primeira situação, os paleocanais não possuem nenhum tipo de cobertura vegetal e são caracterizados nas imagens por elevados valores de brilho (i.e., reflectância dos pixels) nas bandas do infravermelho (Fig. 2a). Paleocanais com algum tipo de cobertura vegetal possuem um único pico de reflectância no infravermelho próximo (Figs. 2a/c). Nestes casos, a intensidade do brilho está em função do tipo e volume da vegetação, sendo que no geral espécies de menor porte possuem mais brilho.

As técnicas existentes para extrair os paleocanais das imagens orbitais podem ser divididas entre as que utilizam métodos de mapeamento semi-automáticos e aquelas que fazem uso da análise visual. Métodos de mapeamento semi-automáticos exigem menor intervenção humana e são realizados com auxílio da coleta de amostras digitais, identificadas nas imagens e representativas dos paleocanais. Segundo Mather (2004), os algoritmos mais utilizados para este procedimento são os da Máxima Verossimilhança e Paralelepípedo, também conhecidos como classificadores supervisionados. Possuem a vantagem de extrair informações detalhadas para vastas áreas, em um menor espaço de tempo que os outros métodos. No entanto, a variabilidade espectral de um mesmo alvo, como é o caso para os paleocanais (Fig. 2), comumente resulta na produção de resultados equivocados. Neste sentido, a análise visual pode contornar as deficiências dos métodos semi-automáticos, pois depende apenas da capacidade do intérprete de identificar e extrair as feições de interesse.

MATERIAL E MÉTODOS O mapeamento dos paleocanais no megaleque do rio Taquari foi realizado empregando-se dados e métodos de sensoriamento remoto. Para correta interpretação dos resultados, é importante descrever as características das imagens, assim como a detalhar das etapas e processamentos utilizados. A seguir são apresentadas as especificidades técnicas dos dados de sensoriamento remoto, métodos empregados e a forma de análise dos resultados.

Dados de sensoriamento remoto Dados de sensoriamento remoto podem ser classificados em função da região do espectro eletromagnético que os sensores operam. Neste sentido, existem três regiões bem definidas: A) óptica, comprimentos de onda de 0,4 a $3 \mu \mathrm{m}$; B) termal, comprimentos de onda de 3 a $15 \mu \mathrm{m}$ e; C) microondas, comprimentos de onda de 0,01 a $100 \mathrm{~cm}$. Neste artigo foram utilizados somente dados ópticos, coletados pelo Advanced Spaceborne Thermal Emission and Reflection Radiometer (ASTER).

O sensor ASTER está a bordo do satélite EOS/ Terra, sendo composto por três instrumentos imageadores: VNIR, que opera na região do visível e infravermelho próximo $(0,52$ a $0,86 \mu \mathrm{m})$; SWIR, que opera na região do infravermelho de ondas curtas $(1,60$ a $2,43 \mu \mathrm{m})$ e; TIR, que adquiri imagens no infravermelho termal 
$(8,12$ a $11,65 \mu \mathrm{m})$. Foram utilizados dados coletados pelo instrumento VNIR, devido ao fato de apresentar melhor resolução espacial que os demais componentes $(15 \mathrm{~m})$, e por conter as bandas espectrais ideais para a identificação dos paleocanais. Estas imagens foram adquiridas pelo Land Processes Distributed Active Archive Center (LP-DAAC), com alto nível de processamento (L3). Este tipo de produto possui exatidão geométrica de $\pm 0,1$ pixel, ou seja, superior a $2 \mathrm{~m}$ (Abrams \& Hooke 2002). E corrigido para possíveis interferências da atmosfera e convertido para valores de reflectância, com exatidão absoluta de $\pm 0,01 \%$ para alvos com reflectância inferior a $15 \%$, e $\pm 7 \%$ para alvos com reflectância superior a $15 \%$ (Abrams \& Hooke 2002). Foram necessárias um total de 34 cenas para cobrir todo o megaleque do Taquari, adquiridas entre os anos de 2001 e 2006, preferencialmente entre os períodos de estiagem.

Mapeamento dos paleocanais Para realçar os depósitos de paleocanais aplicou-se em todas as imagens a técnica de realce por decorrelação. Este processamento remove a correlação entre as bandas espectrais e aumenta a variância das composições coloridas (Mather 2004). Na prática produz uma imagem com cores altamente saturadas e maior contraste que uma composição colorida normal, permitindo melhor identificação dos paleocanais (Fig. 3).

O método utilizado para o mapeamento dos paleocanais foi baseado na análise visual das imagens processadas. Desta maneira, as feições foram identificadas e extraídas manualmente no sistema de informação geográfica (SIG) ArcGIS 9.2, conforme descrito em Zani (2008). Para a sistematização desta tarefa, a área do megaleque foi subdividida em quadrículas regulares com articulação compatível com a escala 1:50.000 do IBGE (Fig. 4). Este procedimento também visou di-
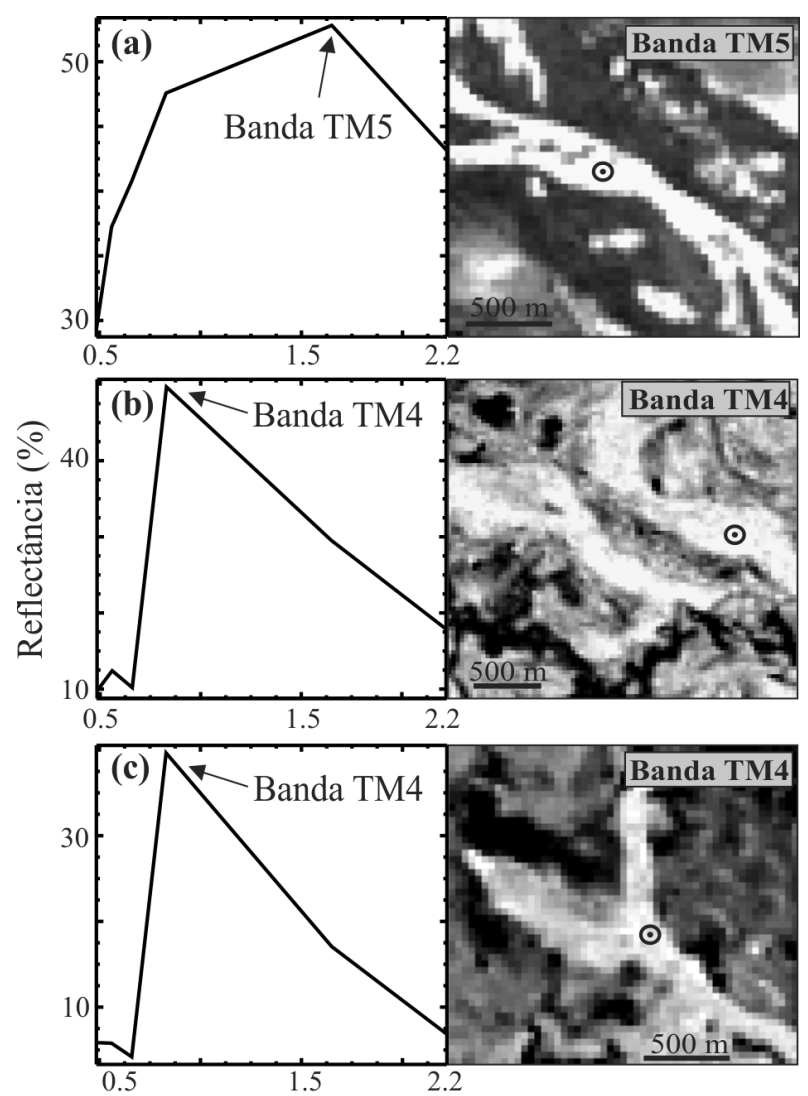

Comprimento de onda $(\mu \mathrm{m})$

Figura 2 - Comportamento espectral dos paleocanais. Os espectros de reflectância estão amostrados de acordo com o intervalo das bandas do sensor TM/Landsat. a) Depósitos arenosos expostos; b) Parcialmente cobertos por vegetação rasteira; c) Cobertos por vegetação arbustiva.
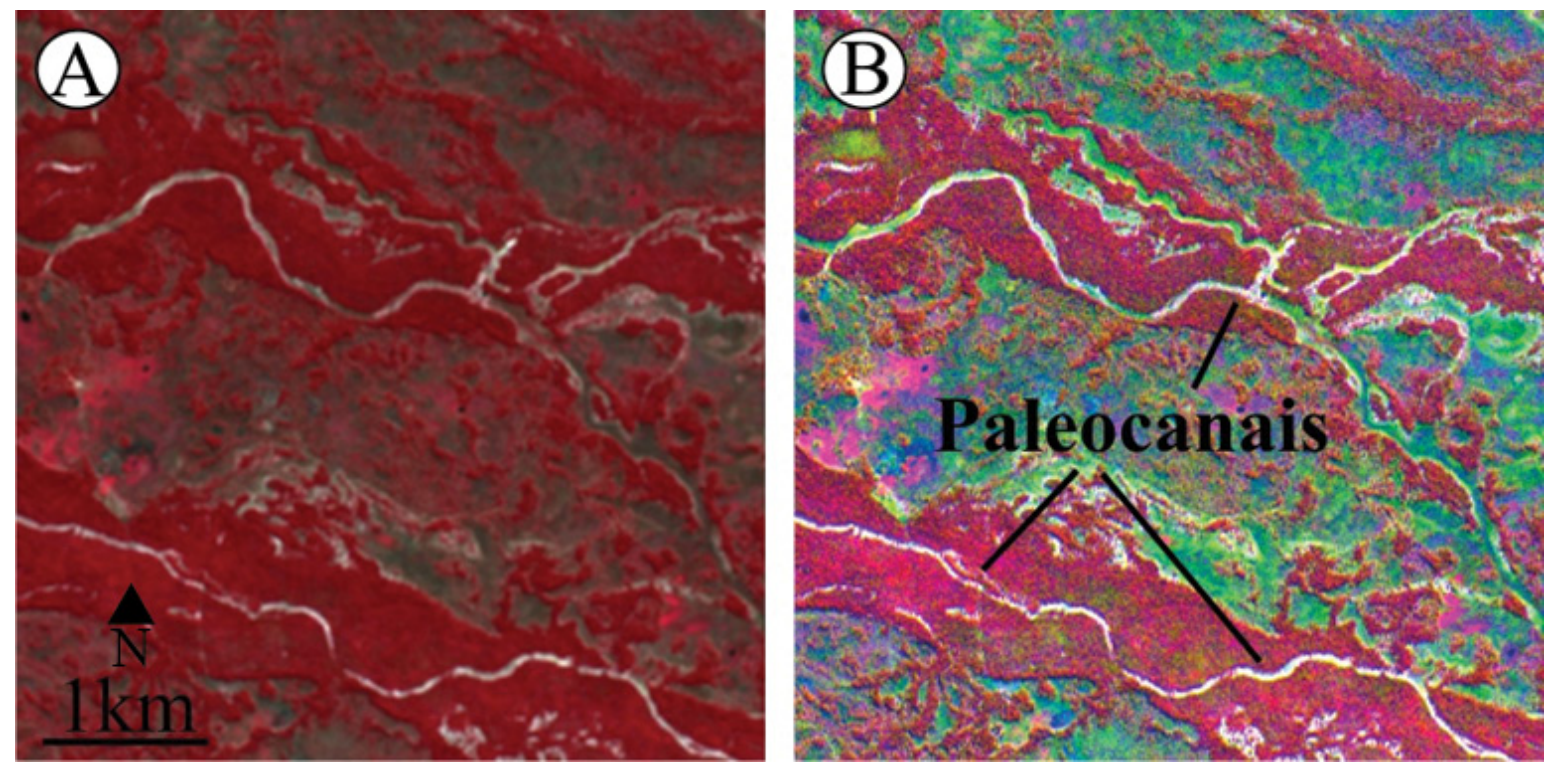

Figura 3 - Composição colorida ASTER 3(r)2(g)1(b) original (A) e após o processamento de realce por decorrelação $(B)$. 
minuir a subjetividade inerente ao método de análise visual.

A partir dos vetores que representam os paleocanais cartografados, foi calculada a densidade de drenagem $\left(\mathrm{D}_{\mathrm{d}}\right)$ total (Horton 1945) (equação 1):

$D_{d}=\frac{2 L}{A_{d}}$

sendo, $\Sigma L$ a somatória do comprimento de todos paleocanais em uma área $A_{d}$. Para a representação espacial deste parâmetro, definiu-se $\mathrm{A}_{d}$ como uma célula de $500 \mathrm{~m}^{2}$, codificada em função do comprimento total de paleocanais, expressa em unidades de $\mathrm{m} . \mathrm{m}^{-2}$. Dados sobre as orientações dos paleocanais foram obtidas com auxílio de gráficos de rosetas, que destacam os azimutes de maior frequência extraídos dos vetores cartografados. Estas informações foram comparadas com os lobos deposicionais identificados por Assine (2003) e subsidiaram interpretações sobre a dinâmica geomorfológica do megaleque.

RESULTADOS E DISCUSSÃO Foram cartografados um total de $33.205 \mathrm{~km}$ de paleocanais, além de 813 $\mathrm{km}$ de canais distributários ativos e a calha atual do rio Taquari (Fig. 5). Esta vasta rede testemunha o caráter mutante do rio Taquari, responsável pelos processos de transporte de sedimentos para o megaleque, que segundo Ab'Saber (1988) ocorrem desde o Pleistoceno tardio. Os paleocanais possuem dominantemente padrão do tipo distributário, caracterizado pela bifurcação dos canais e perda de vazão à jusante. Esta configuração de drenagem é comumente observada em ambientes

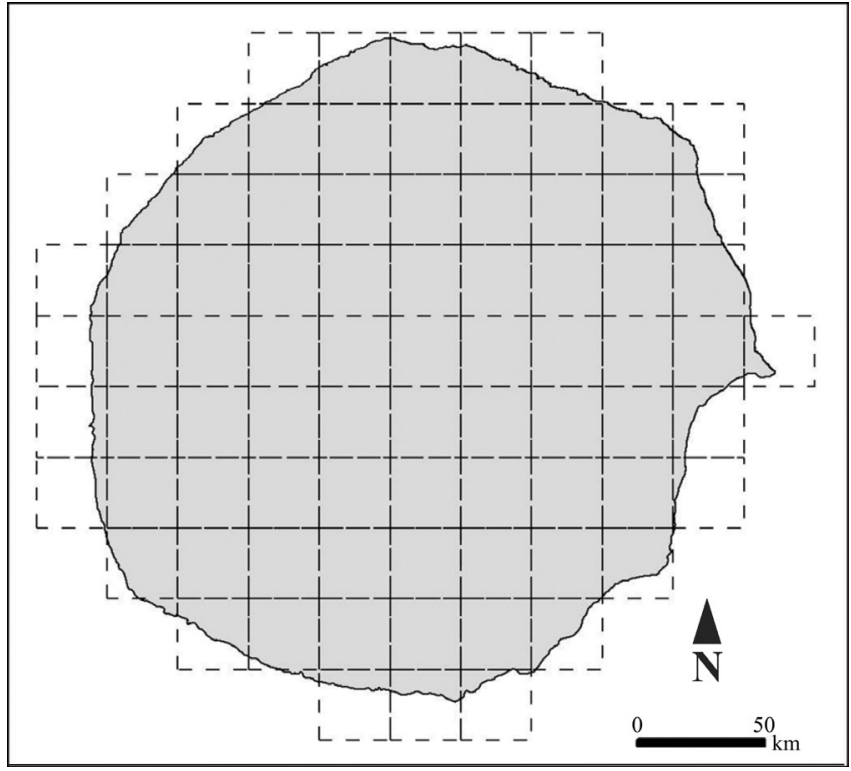

Figura 4 - Área do megaleque do rio Taquari subdividida em folhas topográficas compativeis com a escala 1:50.000.

de megaleques (Weissmann et al. 2010), e constituem a unidade básica que formam os lobos deposicionais (Zani et al. 2009b). Na porção sul do megaleque, conjuntos de paleocanais orientados $45^{\circ} \mathrm{NE}$ evidenciam o controle estrutural exercido pelo Lineamento Transbrasiliano na Bacia do Pantanal, assim como observado por Assine \& Soares (2004).

Em drenagens tributárias, o parâmetro densidade de drenagem expressa o grau de dissecação da su-

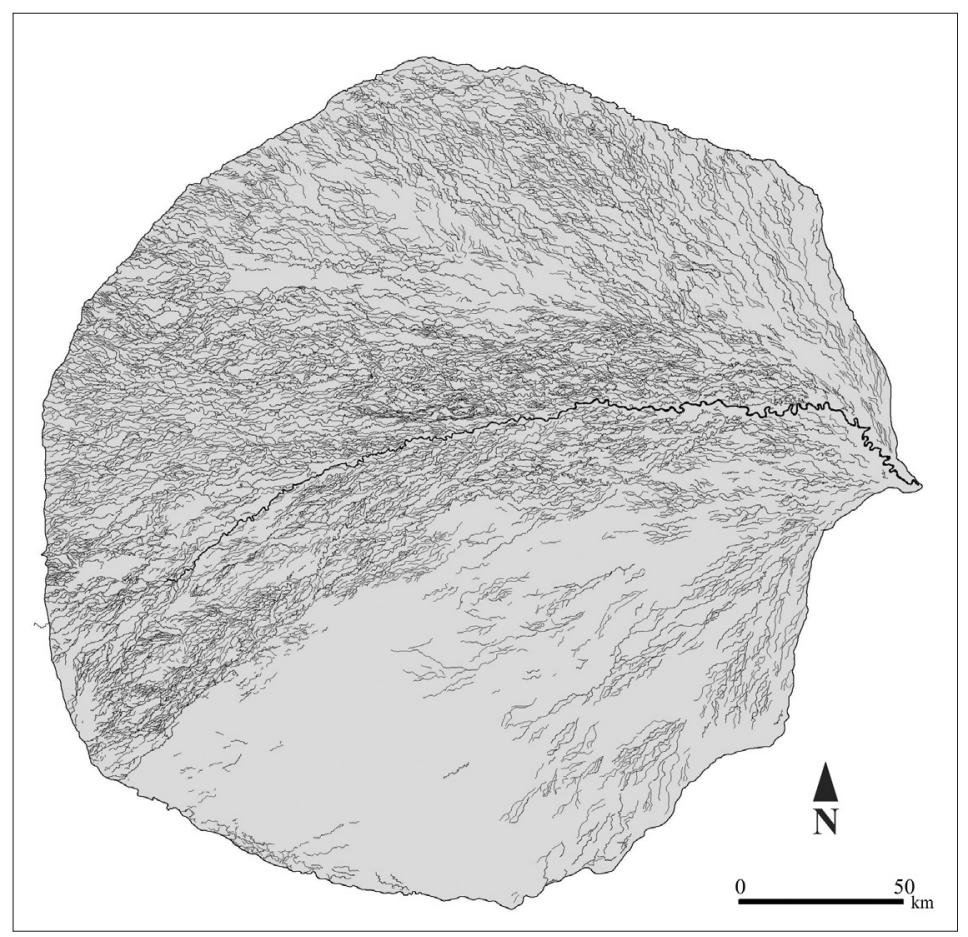

Figura 5 - Rede de paleocanais e drenagem atual cartografada. 
perfície (Knighton 1998) e reflete a interação entre os processos de incisão que formam os canais e a resistência do substrato. No caso de paleocanais, este parâmetro pode estar associado com o estado de preservação destas feições na superfície. Verifica-se zonas com diferentes densidades de paleocanais no megaleque (Fig. 6). As plesmente um fenômeno autocíclico do sistema fluvial, sem nenhuma causa evidente (Wells \& Dorr 1987).

As orientações dos paleocanais são totalmente concordantes com a distribuição dos lobos deposicionais propostos por Assine (2003; Fig. 8). Devido à baixa densidade de feições mapeadas na Nhecolância (lobo 6), não

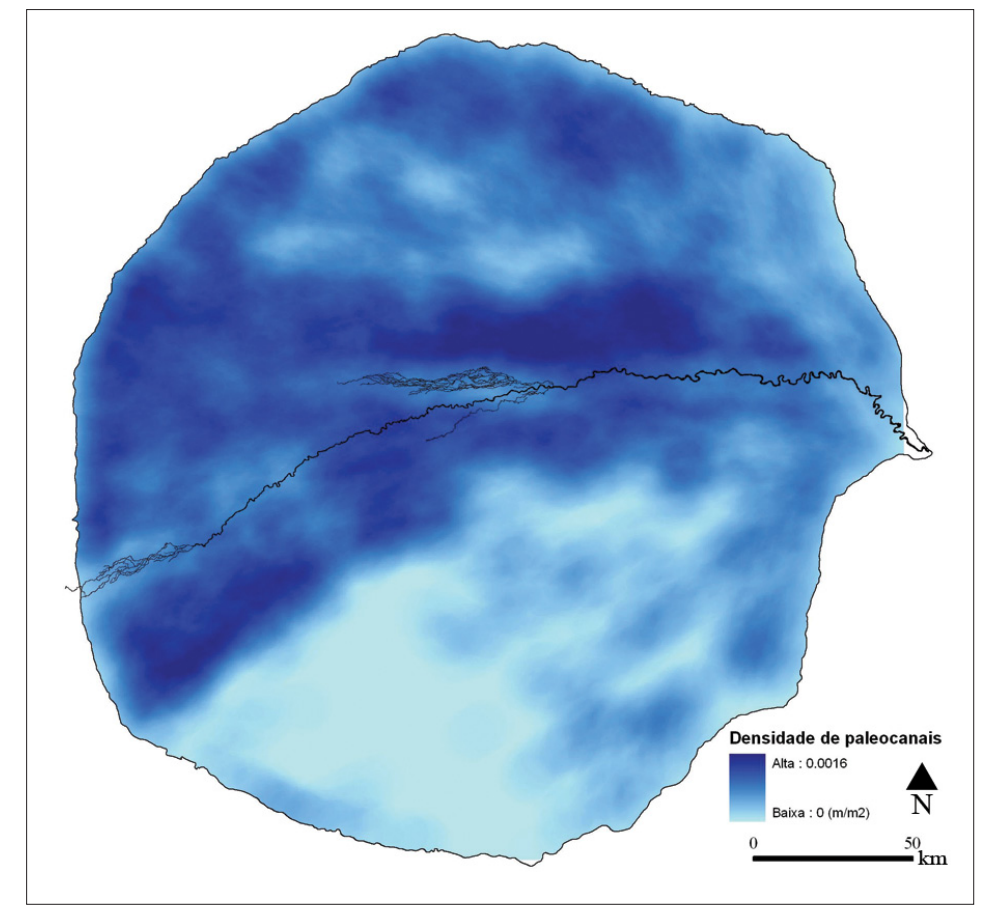

Figura 6 - Densidade espacial dos vetores que representam os paleocanais. Estão sobrepostos os canais distributários atuais e o rio Taquari.

maiores densidades estão próximas ao canal atual do rio Taquari, ao passo que as menores estão na porção sul do megaleque, na região conhecida por Nhecolândia. Assim, nos compartimentos mais antigos, como é o caso da Nhecolândia (Ab'Saber 1988, Assine 2005), os paleocanais estão menos preservados. Esta relação também pode ser observada nos demais lobos deposicionais, sugerindo que o parâmetro densidade seja proporcional à idade relativa dos lobos deposicionais (Fig. 7).

Os paleocanais cartografados sugerem que avulsões fluviais no rio Taquari ocorrem em distintas magnitudes. Avulsões de caráter local são mais comuns e afetam apenas os canais distributários de menor ordem. Avulsões de grande magnitude alteram o curso principal do rio, sendo responsáveis pela dinâmica de construção e abandono dos lobos deposicionais. Os recentes processos de avulsão que ocorreram no rio Taquari, documentados por Padovani et al. (2001) e Assine (2005), são de média magnitude. Segundo Slingerland \& Smith (2004), o tempo de recorrência para eventos de grande magnitude em rios aluviais, como o Taquari, pode variar de 30 a 1000 anos. Pesquisas em outras áreas sugerem que as avulsões podem estar relacionadas com terremotos (Jain \& Sinha 2003), eventos extremos de cheia (Leeder 1999), ou sim-

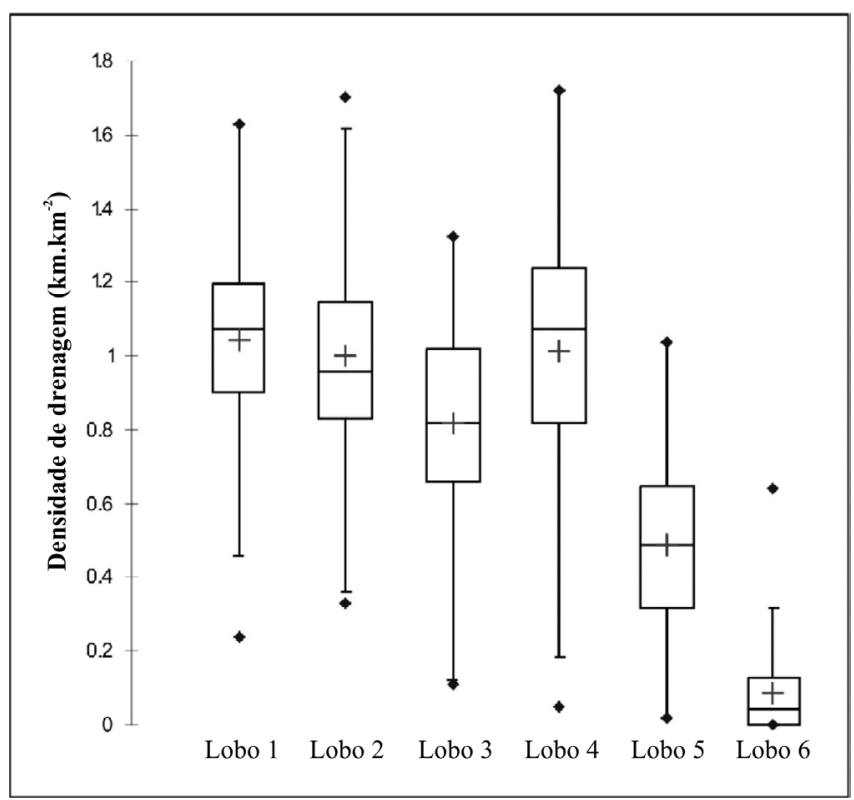

Figura 7 - Distribuições dos valores de densidade de drenagem para os lobos deposicionais. 


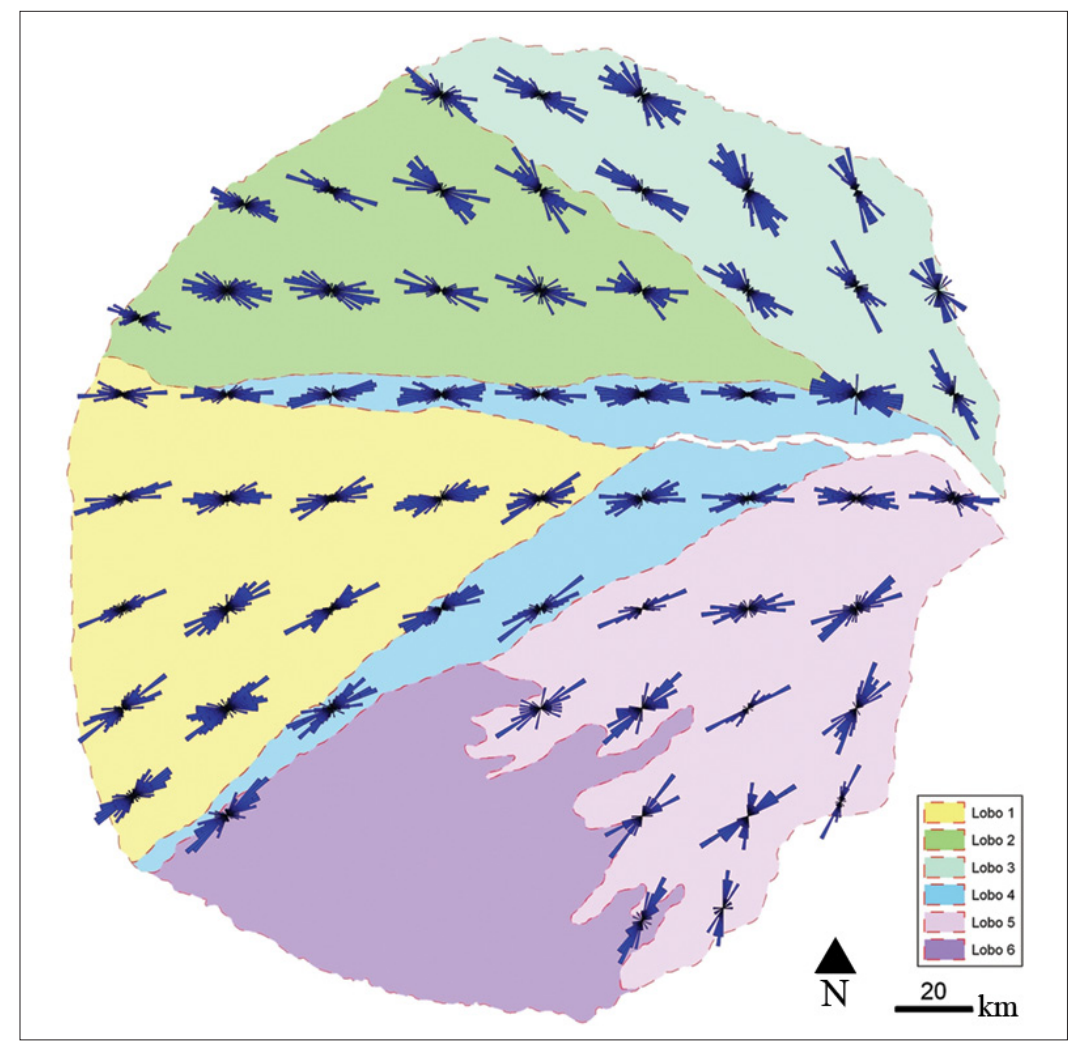

Figura 8 - Gráficos de roseta com as orientações da drenagem extraída, sobrepostos aos lobos deposicionais.

foi possível obter os paleofluxos para esta região. No entanto, as orientações das lagoas existentes (Tricart 1982, Klammer 1982, Zani 2008) são semelhantes aos paleocanais do lobo 5, sugerindo que feições relictas como as existentes na Nhecolândia podem ter condicionado a drenagem do megaleque. Observa-se que a distribuição dos gráficos de roseta (Fig. 8) evidencia o padrão distributário dos paleocanais, sendo que e as diferenças nas orientações sustentam que os lobos deposicionais representam distintos eventos de sedimentação.

CONCLUSÕES Este trabalho apresentou o primeiro mapeamento sistemático dos paleocanais que estão preservados na superfície do megaleque do Taquari. Através deste plano de informação foi possível constatar que: 1) redes de drenagem distributárias constituem as unidades básicas dos lobos deposicionais, corroborando com Zani et al. (2009b); 2) a densidade dos paleocanais pode estar relacionada com a idade relativa dos lobos deposicionais, sendo que os mais recentes possuem maior densidade; 3) antigos fenômenos de avulsão do rio Taquari estão registrados por paleocanais, sendo que os de pequena magnitude são mais frequentes.

Os vetores que representam os paleocanais obtidos neste trabalho constituem um tipo de informação básica, que é de grande importância para outros tipos de estudos ambientais no megaleque do Taquari. Pretendese utilizar estes dados para refinar o modelo digital de elevação (MDE) gerado pela Shuttle Radar Topography Mission (SRTM), a fim de gerar um produto adequado para a análise dos atributos regionais do relevo. Este plano de informação também será utilizado para caracterização quantitativa da drenagem, que por meio de índices morfométricos, pode sinalizar áreas do megaleque que estão sob maior influência de movimentos tectônicos recentes.

Agradecimentos Os autores externam seus agradecimentos à FAPESP pelo apoio à pesquisa (processo 07/55987-3) e concessão de bolsa de mestrado a Hiran Zani (processo 06/02381-8), ao CNPq pela concessão de bolsa PQ a Mario L. Assine (308724/2006-2), ao revisor da RBG que contribuiu para o aprimoramento deste trabalho e ao editor associado Elvo Fassbinder pelo cuidado na editoração do artigo.

\section{Referências}

Ab'Saber A.N. 1988. O Pantanal Matogrossense e a teoria dos refúgios. Rev. Bras. Geog., 2:9-57,

Abrams M \& Hook S. 2002. ASTER user handbook. Pasadena:
Jet Propulsion Laboratory, sem paginação.

Assine M.L. 2003. Sedimentação na Bacia do Pantanal matogrossense, centro oeste do Brasil. Rio Claro, Tese de Livre 
Docência, Universidade Estadual Paulista Júlio de Mesquita Filho, 105 p.

Assine M.L. \& Soares P.C. 2004. Quaternary of the Pantanal, west-central Brazil. Quaternary International, 114:23-34.

Assine M.L. 2005. River avulsions on the Taquari megafan, Pantanal wetland, Brazil. Geomorphology, 70:357-371.

Braun E.H.G. 1977. Cone aluvial do Taquari, unidade geomórfica marcante na planície quaternária do Pantanal. Rev. Bras. Geog., 34:164-180.

Horton R. 1945. Erosional development of streams and their drainage basins: hydrophysical approach to quantitative morphology. Bulletin of the Geological Societyof America, 56:275-370

Jain V. \& Sinha R. 2003. Hyperavulsive-anabranching Baghmati river system, north Bihar plains, eastern India. Zeitschrift fur Geomorphologie, 47:106-116.

Jones H \& Hajek E. 2007. Characterizing avulsion stratigraphy in ancient alluvial deposits. Sedimentary Geology, 202:124-137.

Knighton D. 1998. Fluvial forms and processes; a new perspective. Oxford, Hodder Arnold, $400 \mathrm{p}$.

Klammer G. 1982. Die Palaovuste des Pantanal von mato grosso und die pleistozane Klimageschichte der brasilianischen Randtropen. Zeitschrift fur Geomorphologie, 26:393-416.

Leeder M. 1999. Sedimentology and sedimentary basins: from turbulence to tectonics. Nova York, John Wiley, 608 p.

Mather P. 2004. Computer processing of remotely-sensed images. Nova York, John Wiley, $324 \mathrm{p}$.

Padovani C., Pontara R., Pereira J. 2001. Mudanças recentes de leito no baixo curso do rio Taquari, no Pantanal matogrossense. Boletim Paranaense de Geociências, 49:33-38.

Ramasamy S.M, Bakliwal P. C., Verma R.P. 1991. Remote sensing and river migration in Western India. International Journal of Remote Sensing, 12:2597-2609.

Sinha R. 1996. Paleohydrology of Quaternary river systems of north Bihar plains, India. In: Singh V.P. \& Kumar B. (eds.). Surface Water Hydrology. Amsterdan, Kluwer, p. 29-41.

Slingerland R. \& Smith N. 2004. River avulsion and their deposits. Annual Review of Earth and Planetary Sciences, 32:257-285.

Tricart J. 1982. El Pantanal: un ejemplo del impacto geomorfológico sobre el ambiente. Informaciones Geográficas (Chile), 29:81-97.

Weissmann G., Hartley A., Nichols G., Scuderi L., Olson M., Buehler H., Banteah R. 2010. Fluvial form in modern continental sedimentary basins: distributive fluvial systems. Geology, 38:39-42.

Wells N.A. \& Dorr J.N. 1987. Shifting of the Kosi River, northern India. Geology, 15:204-207.

Wray R. 2009. Palaeochannels of the Namoi river floodplain, New South Wales, Australia: the use of multispectral Landsat imagery to highlight a Late Quaternary change in fluvial regime. Australian Geographer, 40:29-49.

Zani H. 2008. Mudanças morfológicas na evolução do megaleque do Taquari: uma análise com base em dados orbitais. Rio Claro, Dissertação de Mestrado, Universidade Estadual Paulista Júlio de Mesquita Filho, 85 p.

Zani H., Assine M.L., Silva A., Corradini F., Kuerten S., Gradella F. 2009a. Geoformas deposicionais e feições erosivas no Pantanal mato-grossense identificadas por sensoriamento remoto. Geografia, 34:643-654.

Zani H., Assine M.L., Silva A., Corradini F. 2009b. Redes de drenagem distributária e formas deposicionais no megaleque do Taquari, Pantanal: uma análise baseada no MDESRTM. Revista Brasileira de Geomorfologia, 10:21-28.

Manuscrito ID 17520

Submetido em 18/05/2010

Aceito em 30 de novembro de 2010 